\title{
Impacto del monitoreo terapéutico de vancomicina y amikacina en la optimización de dosis de antimicrobianos en pacientes pediátricos
}

\section{Impact of the vancomycin and amikacin therapeutic monitoring in the optimization of antimicrobial dose in pediatric patients}

Paula Chávez M. ${ }^{1}$, Aivlys Fernández H. ${ }^{1}$, Rubén Hernández M. ${ }^{2}$ y Luis Delpiano M. ${ }^{3}$

PPediatra. Universidad de Chile.
${ }^{2}$ Químico Farmacéutico. Servicio de Pediatría Hospital Clínico San Borja Arriarán.
${ }^{3}$ Pediatra Infectólogo. Hospital Clínico San Borja Arriarán.

Los autores declaran no haber recibido financiamiento ni presentan conflicto de interés para el desarrollo de esta publicación.

Recibido: 20 de octubre de 2020 / Aceptado: 26 de abril de 2021

\section{Resumen}

Introducción: La monitorización de antimicrobianos mediante sus concentraciones plasmáticas permite determinar la posología óptima de éstos, conducta esencial en pediatría. Objetivos: Describir la monitorización de concentraciones plasmáticas de antimicrobianos y el ajuste de dosis en población pediátrica para determinar si las dosis utilizadas alcanzan rangos terapéuticos. Pacientes y Métodos: Estudio descriptivo, retrospectivo, utilizando una base de datos con medición de concentraciones plasmáticas de amikacina y vancomicina en pacientes pediátricos del Hospital San Borja Arriarán, entre 2015-2018. Se determinó el número de pacientes que alcanzó rango terapéutico con dosis inicial, cuántos requirieron ajuste y sus características. Resultados: Se monitorizó 104 concentraciones totales. Para vancomicina 65 concentraciones plasmáticas eran basales encontrándose fuera de rango terapéutico $56,5 \%$; de los que requirieron ajuste, $25 \%$ fueron neonatos con mayor probabilidad de estar fuera de rango versus otros $(\mathrm{p}=0,022)$. Para amikacina la Cpeak estuvo en rango en $60 \%$ de mediciones; $15,4 \%$ requirió ajuste incluyendo pacientes con fibrosis quística y oncológicos. No fue necesario efectuar ajustes en pacientes sin co-morbilidad. Conclusión: La medición de concentraciones plasmáticas es necesaria para ajustar de forma individualizada la dosis, especialmente en pacientes pediátricos con fibrosis quística, oncológicos y en neonatología, donde es más probable no alcanzar rango terapéutico con las dosis iniciales.

Palabras clave: monitorización terapéutica; vancomicina; amikacina; pediatría.

\section{Abstract}

Background: The monitoring of antimicrobial therapy through plasma levels makes it possible to determine the optimal dosage of antimicrobials, an essential approach in pediatrics. Aim: To describe the monitoring of plasma antimicrobial levels and dose adjustment in the pediatric population to determine if the doses used reach therapeutic ranges. Methods: Retrospective, descriptive study using a database with measurement of plasma levels of amikacin and vancomycin in pediatric patients at San Borja Arriarán Hospital between 2015-2018. The number of patients who reached the therapeutic range with the initial dose, how many required adjustment and their characteristics were determined. Results: 104 total levels were monitored. For vancomycin 65 plasmatic levels were baseline, being outside the therapeutic range $56.5 \% ; 25 \%$ of those requiring adjustment were neonates with a higher probability of being out of range versus others $(\mathrm{p}=0.022)$. For amikacin, Cpeak was in range in $60 \%$ of measurements; $15.4 \%$ required adjustment, including patients with cystic fibrosis and cancer, without adjustments in patients without comorbidity. Conclusion: Measurement of plasma levels is necessary to individually adjust the dose, especially in pediatric patients with cystic fibrosis, oncology and in neonatology where it is more likely not to reach a therapeutic range with initial doses.

Keywords: therapeutic monitoring; vancomycin; amikacin; pediatrics. 


\section{Introducción}

L os antimicrobianos están entre los medicamentos más recetados en niños; aproximadamente $20 \%$ de las consultas ambulatorias y $60 \%$ de los pacientes hospitalizados tiene indicado al menos un antimicrobiano ${ }^{1}$. La dosificación suele ser por kilogramo de peso y extrapolada de dosis de adulto, pudiendo llevar a sobre o subdosificación ${ }^{2}$.

El mal uso de antimicrobianos se ha relacionado con aumento de morbi-mortalidad y emergencia de resistencia bacteriana, por lo que desde sociedades científicas se fomentan los programas de monitorización terapéutica a través de medición de sus concentraciones plasmáticas como herramienta esencial para optimizar su uso, basados en modelos farmacocinéticos (PK) y farmacodinámicos (PD). Éstos describen la relación entre concentración monitorizada, efecto bactericida y/o capacidad de inhibir el crecimiento del patógeno (concentración inhibitoria mínima o CIM), estableciendo tres modelos: dependientes de tiempo (por ej.: $\beta$-lactámicos), de concentración (por ej.: aminoglucósidos) y área bajo la curva dependientes (ABC) (por ej.: vancomicina). La monitorización terapéutica consiste en medir la concentración plasmática del fármaco permitiendo reducir toxicidad y maximizar eficacia antimicrobiana ${ }^{3}$. Los resultados de esta monitorización deben ser interpretados cuidadosamente y el ajuste de dosis realizado por un equipo multidisciplinario ${ }^{4}$, logrando mejorar el manejo clínico y establecer modelos PK poblacionales pediátricos que guíen la optimización de antimicrobianos como aminoglucósidos y vancomicina, considerando que en pacientes pediátricos existen grandes diferencias en la absorción, distribución, metabolismo y eliminación de fármacos respecto a los adultos; incluso, dentro de esta misma población es posible observar diferencias, según edad y/o presencia de comorbilidades.

Optimizar la dosis de vancomicina es difícil en niños; en el año 2009 la Sociedad Americana de Infectología, la Sociedad de Farmacéuticos Hospitalarios y la Sociedad de Farmacéuticos en Infectología desarrollaron el primer consenso para la monitorización terapéutica de vancomicina que sugirió el uso de concentraciones plasmáticas valle entre 15 y $20 \mu \mathrm{g} / \mathrm{mL}$ como sustituto para $\mathrm{ABC} /$ CIM > 400 considerada medida ideal en infecciones por Staphylococcus aureus, valor que fuera actualizado en su última versión $2020^{5}$. La mayoría de los estudios disponibles analiza aisladamente la monitorización terapéutica en pacientes críticos hasta establecer la dosis pediátrica recomendada de $60 \mathrm{mg} / \mathrm{kg} /$ día endovenosa que utiliza nuestro centro. Existen grupos de pacientes pediátricos especiales en quienes el uso de vancomicina y su monitorización terapéutica resulta crítica como los recién nacidos $(\mathrm{RN})$, pacientes oncológicos, aquellos con fibrosis quística $(\mathrm{FQ})^{6-8}$ y pacientes con insuficiencia renal, principalmente para evitar nefrotoxicidad.

La razón para monitorizar las concentraciones plasmáticas en aminoglucósidos radica en el estrecho rango terapéutico que determina riesgo de oto y nefrotoxicidad, además de la variación inter e intrasujeto en la PK. La máxima respuesta clínica ocurre con concentraciones plasmáticas máximas o peak mayores que la CIM por un factor de al menos $8^{(2)}$. En niños sólo existe evidencia indirecta de un meta-análisis, que sugiere que una sola dosis diaria, asociada con una concentración máxima mayor tendría mejores tasas de erradicación microbiológica y eficacia comparada con dosis múltiples ${ }^{9}$.

En lo anterior queda expuesta la importancia de la monitorización terapéutica para guiar y optimizar la terapia antimicrobiana en niños, por lo que el objetivo principal de este trabajo es evaluar el impacto de la monitorización de concentraciones plasmáticas de vancomicina y amikacina y eventual ajuste de dosis en población pediátrica del Hospital Clínico San Borja Arriarán (HCSBA), con el fin de determinar si las dosis recomendadas alcanzan rangos terapéuticos y si existen diferencias según grupo etario o comorbilidad.

\section{Pacientes y Método}

La población estudiada correspondió a pacientes bajo 15 años de edad, internados en HCSBA, en unidades pediátricas de sala básica, oncología, paciente crítico pediátrico (UCIP) y neonatología, durante el período 2015 a 2018, a quienes se le indicó tratamiento con amikacina (único aminoglucósido recomendado en las guías institucionales) o vancomicina y se les realizó monitorización terapéutica con técnicas disponibles en nuestro laboratorio. Los datos se obtuvieron de forma retrospectiva desde registros de trabajo de la unidad de infectología pediátrica del HCSBA creada desde el quehacer diario y conjunto entre infectólogos pediatras y farmacéutico clínico, que recopila la información de los pacientes tratados con estos fármacos, los cuales fueron evaluados por el equipo requiriendo toma de concentraciones plasmáticas de antimicrobiano y ajuste de dosis según necesidad. Desde este registro se construyó una base de datos que permitió realizar análisis estadístico descriptivo con información como: edad, género, foco de infección, comorbilidad (FQ, patología oncológica, shock séptico definido como la presencia de sepsis más disfunción cardiovascular, u otra), resultado de cultivos y CIM del patógeno aislado, unidad de hospitalización, antimicrobiano indicado, dosis inicial de tratamiento, tipo (momento) de concentración plasmática, resultado de la concentración plasmática medida y la necesidad de ajuste de dosis o no. Se excluyeron aquellos pacientes con disfunción renal, definida como 


\section{Resultados}

uno o más de los siguientes criterios al momento del
monitoreo: clearance de creatinina $<50 \mathrm{ml} / \mathrm{min} / 1,73 \mathrm{~m}^{2}$ por fórmula de $\mathrm{Schwartz}^{10}$, diagnóstico de insuficiencia renal crónica o necesidad de terapia de reemplazo renal, además, de aquellos con registros incompletos.

Los pacientes incluidos corresponden a pacientes con sospecha clínica o confirmación de infección bacteriana invasora, a excepción de pacientes FQ en quienes se consideraron también muestras bacteriológicas respiratorias.

Para amikacina la monitorización se realizó con concentración peak (medición tras la segunda dosis y media hora posterior al término de la infusión) considerando terapéutico si resultara 10 veces sobre la CIM bacteriana; y/o valle (medición media hora previo a la segunda dosis) siendo la concentración esperada $<1 \mu \mathrm{g} / \mathrm{mL}$. La dosis de amikacina estándar empleada es $15 \mathrm{mg} / \mathrm{kg}$ /día para población pediátrica general; en pacientes oncológicos la dosis corresponde a $20 \mathrm{mg} / \mathrm{kg} /$ día y para pacientes con FQ se indican $30 \mathrm{mg} / \mathrm{kg} / \mathrm{día}^{6}$; para $\mathrm{RN}$ las dosis son extremadamente variables y se administran según edad gestacional y cronológica, de acuerdo al manual Neofax ${ }^{\circledR 11}$.

Para vancomicina la monitorización se realizó mediante la determinación de concentraciones basales o valle (Cbasal), que corresponde a la concentración plasmática medida media hora previa a la cuarta dosis, correspondiente al estado de equilibrio en pacientes sin insuficiencia renal. Se considera terapéutica una concentración entre 10 y $20 \mu \mathrm{g} / \mathrm{mL}$. La dosis estándar en nuestro centro es $60 \mathrm{mg} / \mathrm{kg} /$ día para niños $>1$ mes y para $\mathrm{RN}$ dosis según edad cronológica y gestacional, de acuerdo al manual Neofax ${ }^{\circledR 11}$.

Para el examen no se requiere preparación del paciente, se recolectan $2 \mathrm{ml}$ de sangre en tubo sin anticoagulante, a temperatura ambiente. En el laboratorio, la muestra es analizada mediante técnica de inmunoensayo con el equipo Centaur ${ }^{\circledR} \mathrm{CP}$ para vancomicina y Dimensión ${ }^{\circledR}$ para amikacina, ambos de marca Siemens, validados por el Instituto de Salud Pública. Las concentraciones plasmáticas de amikacina se miden en nuestro laboratorio desde noviembre 2018; previamente las muestras eran enviadas y analizadas en un laboratorio externo.

Los ajustes de dosis fueron realizados por el equipo de infectología pediátrica: infectólogo pediatra y farmacéutico clínico, utilizando el software Precise pk, que utiliza datos farmacocinéticos poblacionales más estadística bayesiana. El análisis de datos se realizó con el software Stata 13 SE, además de la prueba exacta de Fisher para las variables concentración plasmática peak para amikacina y Cbasal para vacomicina, considerando un valor $\mathrm{p}<0,05$ como significancia estadística.

Para este trabajo, se obtuvo autorización del Comité Ético Científico del Servicio de Salud Metropolitano Central. en la Tabla 1.

\section{Monitorización de amikacina}

En el período que contempla esta revisión, se monitorizaron 38 concentraciones plasmáticas de amikacina en una población de 26 pacientes con edades que fluctuaron desde RN hasta 14 años, sin diferencias por sexo. El principal foco que motivó el tratamiento con amikacina fue el foco pulmonar $(73,1 \%$ de casos). De los cultivos realizados previo al inicio del tratamiento $80,8 \%$ de ellos fueron positivos, correspondiendo principalmente a cultivos de secreción bronquial. Tenían diagnóstico de FQ $65,4 \%$ de ellos; $19,2 \%$ de los pacientes no tenían comorbilidad. Se encontraban internados en sala básica el $88,5 \%$. Las características demográficas se detallan

Tabla 1. Características demográficas en población con monitorización de concentraciones plasmáticas de amikacina y vancomicina

\begin{tabular}{|c|c|c|}
\hline & $\begin{array}{c}\text { Amikacina } \\
\text { Total }(\%) \\
\text { ( } \mathrm{n}=26 \text { pacientes })\end{array}$ & $\begin{array}{c}\text { Vancomicina } \\
\text { Total }(\%) \\
\text { (n = } 46 \text { pacientes) }\end{array}$ \\
\hline \multicolumn{3}{|l|}{ Edad (años) } \\
\hline Promedio & 4,8 & 4,1 \\
\hline Rango & $0,02-14$ & $0,014-14$ \\
\hline \multicolumn{3}{|l|}{ Sexo } \\
\hline Masculino & $13(50)$ & $25(54,3)$ \\
\hline Femenino & $13(50)$ & $21(45,7)$ \\
\hline \multicolumn{3}{|l|}{ Foco de infección } \\
\hline Pulmonar & $19(73,1)$ & $9(19,6)$ \\
\hline Sanguíneo & $3(11,5)$ & $13(28,3)$ \\
\hline Renal & $1(3,8)$ & 0 \\
\hline Sin foco & $3(11,5)$ & $13(28,3)$ \\
\hline Cutáneo & 0 & $4(8,7)$ \\
\hline Otros & 0 & $\begin{array}{c}\text { Osteoarticular } 3(6,5) \\
\text { SNC } 3(6,5) \\
\text { Abdominal } 1(2,2)\end{array}$ \\
\hline \multicolumn{3}{|l|}{ Co-morbilidad } \\
\hline Fibrosis quística & $17(65,4)$ & $5(10,9)$ \\
\hline Oncológica & $4(15,4)$ & $7(19,6)$ \\
\hline Sin comorbilidad & $5(19,2)$ & $32(69,6)$ \\
\hline Shock séptico & 0 & $2(4,3)$ \\
\hline \multicolumn{3}{|c|}{ Unidad de hospitalización } \\
\hline Neonatología & $1(3,8)$ & $8(17,4)$ \\
\hline Sala básica & $23(88,5)$ & $22(47,8)$ \\
\hline $\mathrm{UCl}$ & $2(7,7)$ & $16(34,8)$ \\
\hline \multicolumn{3}{|l|}{ Cultivos } \\
\hline Positivos & $21(80,8)$ & $24(52,1)$ \\
\hline Negativos & $5(19,2)$ & $22(47,8)$ \\
\hline
\end{tabular}


De las 38 concentraciones plasmáticas de amikacina, 25 fueron mediciones peak con un promedio de 44,2 $\mu \mathrm{g} /$ $\mathrm{mL}$ y un rango entre 18,2 y $80 \mu \mathrm{g} / \mathrm{mL}$. De éstos, $60 \%$ fueron valores 10 veces sobre la CIM, considerado terapéutico, de los cuales $73,3 \%$ eran pacientes con FQ y $26,7 \%$ otro tipo de pacientes con un valor $\mathrm{p}=1$.

Se monitorizaron 13 concentraciones plasmáticas valle, $84,6 \%$ fue $<0,8 \mu \mathrm{g} / \mathrm{mL}$, con dos concentraciones plasmáticas valle sobre rango terapéutico, con un máximo de $2 \mu \mathrm{g} / \mathrm{mL}$ en una paciente que no recibió ajuste porque el valor se recibió posterior al fin del tratamiento y una concentración plasmática valle de $1,2 \mu \mathrm{g} / \mathrm{mL}$ sin ajuste, dada la CIM bacteriana.

Según las concentraciones plasmáticas medidas, la conducta terapéutica adoptada en $84,6 \%$ de casos $(n=22)$ fue mantener igual dosis; de los cuatro pacientes en que se realizó ajuste, tres tenían diagnóstico de FQ (Figura 1), todos estaban hospitalizados en sala básica.

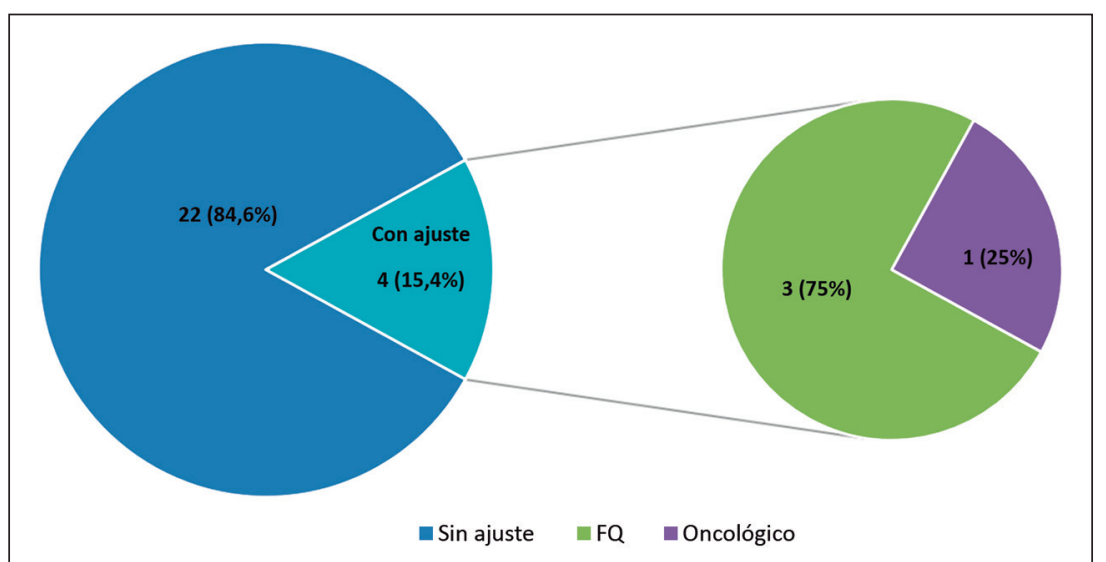

Figura 1. Ajuste de dosis según concentraciones plasmáticas peak de amikacina.

Tabla 2. Descripción de los pacientes tratados con amikacina, dosis de inicio tratamiento y necesidad de ajuste de dosis

\begin{tabular}{|lccc|}
\hline Tipo de paciente & Total $(\%)$ & Dosis $(\mathbf{m g} / \mathbf{k g} /$ día $)$ & Ajuste \\
\hline UCIP & $2(7,7)$ & 15 & 0 \\
\hline Neonatología & $1(3,8)$ & 15 & 0 \\
\hline Sala básica sin comorbilidad & $2(7,7)$ & 15 & 0 \\
\hline Oncológicos & $4(15,4)$ & 20 & $1^{*}$ \\
\hline FQ & $17(65,4)$ & & \\
& 14 & 30 & $2^{* *}$ \\
& 1 & 20 & 0 \\
\hline
\end{tabular}

*Concentración peak 18,5 $\mu \mathrm{g} / \mathrm{mL}$, se aumentó la dosis. **Concentración peak 57,3 y $80 \mu \mathrm{g} / \mathrm{mL}$, en ambos se disminuyó la dosis. ***Concentración peak de $18,2 \mu \mathrm{g} / \mathrm{mL}$, se aumentó la dosis.
Evaluando la dosis utilizada, tanto los pacientes de UCIP, neonatología y sala básica sin comorbilidad iniciaron tratamiento con amikacina $15 \mathrm{mg} / \mathrm{kg} /$ día, sin requerir ajuste de dosis; los pacientes oncológicos iniciaron con 20 $\mathrm{mg} / \mathrm{kg} /$ día, requiriendo ajuste sólo en un caso; mientras que en los pacientes con FQ se utilizaron distintas dosis entre 15 y $30 \mathrm{mg} / \mathrm{kg} /$ día cuyo promedio fue $27,6 \mathrm{mg} /$ $\mathrm{kg}$ /día, requiriendo ajuste en tres ocasiones (Tabla 2), debiendo disminuir dosis en dos de los pacientes con dosis $30 \mathrm{mg} / \mathrm{kg} /$ día y en una oportunidad aumentar dosis en tratamiento iniciado con $15 \mathrm{mg} / \mathrm{kg} /$ día.

\section{Monitorización de vancomicina}

Para la monitorización de vancomicina, se determinaron 66 concentraciones plasmáticas en una población de 46 pacientes, con edades entre RN y 14 años. Las infecciones sin foco y las bacteriemias fueron los principales motivos de tratamiento con este antimicrobiano. De los cultivos realizados 52,1\% fueron positivos, siendo Staphylococcus coagulasa negativa el principal agente, todos con una CIM $=1$. De los pacientes $69,6 \%$ no tenían comorbilidades, $19,6 \%$ eran oncológicos, 10,9\% FQ y 4,3\% tuvieron shock séptico. Respecto al lugar de hospitalización 47,8\% se encontraba en sala básica, 34,8\% en UCIP y $17,4 \%$ en neonatología (Tabla 1 ).

De las 66 mediciones de concentraciones plasmáticas, 65 muestras correspondían a Cbasal con un mínimo de $1,06 \mu \mathrm{g} / \mathrm{mL}$, un máximo de $69,4 \mu \mathrm{g} / \mathrm{mL}$ y un promedio de $14,2 \mu \mathrm{g} / \mathrm{mL}$. Se controló concentración plasmática peak $(27,9 \mu \mathrm{g} / \mathrm{mL})$ en sólo un paciente que estaba hospitalizado en UCIP y tenía FQ. La Cbasal máxima correspondió a paciente oncológico con neutropenia febril de alto riesgo (NFAR) que presentó falla renal aguda intratratamiento.

De las 65 Cbasal, 46 fueron determinaciones iniciales y 19 controles. La Cbasal promedio en determinación inicial fue $14,3 \mu \mathrm{g} / \mathrm{mL}$ con un rango entre $1,06 \mu \mathrm{g} / \mathrm{mL}$ (en paciente $\mathrm{RN}$ ) y $69,4 \mu \mathrm{g} / \mathrm{mL}$ (en paciente previamente mencionado con NFAR), requiriendo ajuste de dosis en 24 pacientes (52,2\%) (Figura 2). Cuatro de ellos (8,7\%) requirieron más de un ajuste para lograr el valor objetivo: dos eran RN (un paciente con endocarditis bacteriana requirió tres ajustes), uno de UCIP con shock séptico y uno de sala básica con artritis séptica.

De los 24 pacientes que requirieron ajuste en la dosificación de vancomicina, $25 \%$ eran $\mathrm{RN}, 25 \%$ pacientes de pediatría general, 20,8\% NFAR, 20,8\% pacientes de UCIP y sólo $8,4 \%$ presentaba FQ; lo que significa que 54,2\% $(n=13)$ de los pacientes que requirieron ajuste de dosis presentaban alguna comorbilidad o eran RN (Gráfico 2). El ajuste de dosis fue variable considerando cada caso en particular, logrando Cbasal en rango terapéutico en todos los pacientes.

De las 46 Cbasal inicial, 43,5\% se encontraban en rango terapéutico con una dosis promedio de vancomicina 
de $45,8 \mathrm{mg} / \mathrm{kg} /$ día. En 41,3\% hubo concentración bajo rango con dosis promedio $58,3 \mathrm{mg} / \mathrm{kg} /$ día y $15,2 \%$ con concentraciones plasmáticas supraterapéuticas utilizando dosis promedio de $52,7 \mathrm{mg} / \mathrm{kg} /$ día.

De los 19 pacientes bajo el rango terapéutico, ocho eran pacientes pediátricos sin comorbilidad, seis eran $\mathrm{RN}$, cuatro pacientes oncológicos y uno con diagnóstico FQ. Ningún RN tuvo Cbasal inicial entre 10-20 $\mu \mathrm{g} /$ $\mathrm{mL}$, comparados con 14 de pediatría, cuatro pacientes de oncología y dos pacientes $\mathrm{FQ}$, con un $\mathrm{p}=0,022$. Al efectuar este mismo análisis con pacientes oncológicos versus otro tipo de pacientes en relación a probabilidad de alcanzar concentraciones plasmáticas terapéuticas, no se logra establecer asociación con un $\mathrm{p}=1$. De los siete pacientes que tuvieron Cbasal sobre rango terapéutico, tres pacientes eran de pediatría general, dos $\mathrm{RN}$, un paciente oncológico y un paciente con FQ.

De los 46 pacientes que recibieron vancomicina $10,9 \%$ tenían como comorbilidad FQ; la dosis inicial en ellos fue $60 \mathrm{mg} / \mathrm{kg} /$ día, excepto en dos en quienes se utilizó otra dosis (50 y $75 \mathrm{mg} / \mathrm{kg} /$ día), requiriendo ambos un ajuste, mientras que ninguno de los que iniciaron tratamiento con $60 \mathrm{mg} / \mathrm{kg} /$ día lo requirió.

\section{Discusión}

Amikacina como antimicrobiano representante de los aminoglucósidos, es uno de los más utilizados para infecciones graves causadas por bacilos gramnegativos ${ }^{12}$; en nuestra serie, este medicamento fue indicado principalmente para manejo de infecciones pulmonares en pacientes FQ, grupo particular que concentró $65,4 \%$ del total de tratamientos con amikacina y $75 \%$ de los ajustes de dosis, lo que apunta a su frecuente indicación para el manejo de exacerbaciones infecciosas pulmonares en estos pacientes con dosis mayores a las habituales y la necesidad, entonces, de monitorizar sus concentraciones plasmáticas. En una revisión sobre $\mathrm{PK} / \mathrm{PD}$ de pacientes con $\mathrm{FQ}^{6}$, destaca la necesidad de monitorizar sus concentraciones plasmáticas debido a la alteración en la distribución del fármaco por hipoalbuminemia e hipogamaglobulinemia además de disminución en la vida media de amikacina. Por otro lado, y conociendo la alta frecuencia de exacerbación pulmonar por Pseudomonas aeuruginosa, y donde sólo 40\% de la concentración sanguínea del antimicrobiano alcanza al pulmón, surge la necesidad de emplear dosis más altas que en la población general. En nuestros pacientes con FQ en que se inició tratamiento con dosis mayores a lo habitual, sólo 17,6\% requirió ajuste; cabe destacar que de los bacilos gramnegativos aislados en este grupo, 53,8\% tuvo CIM $>2 \mu \mathrm{g} / \mathrm{mL}$, lo que permite concluir que de haber iniciado terapia con dosis habituales habría sido necesario un mayor porcentaje de ajuste.

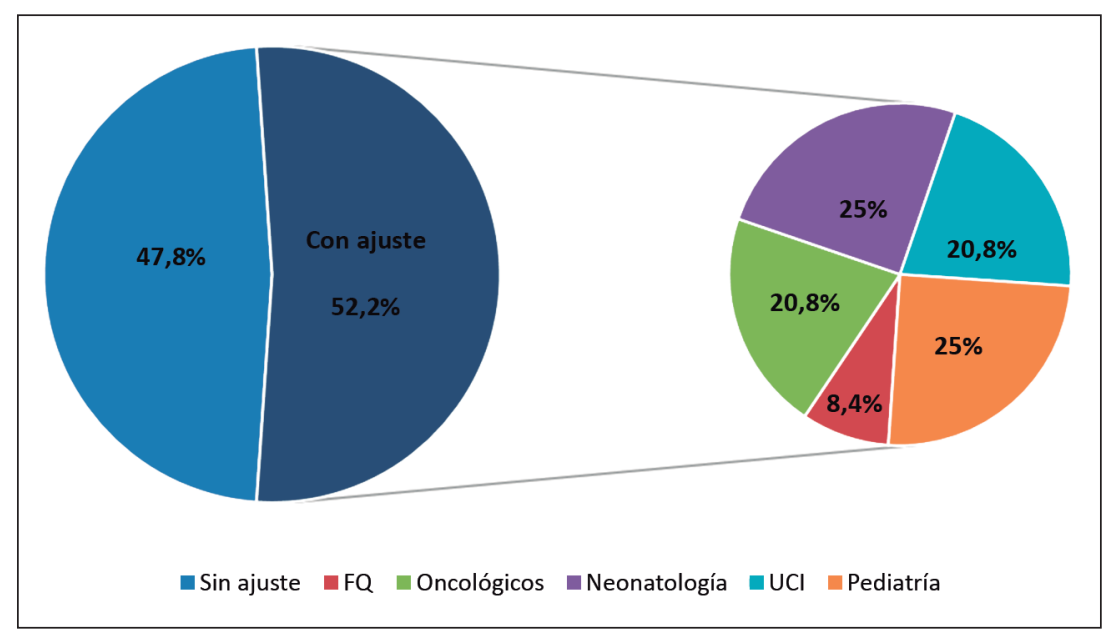

Figura 2. Ajuste de dosis según primera concentración valle vancomicina.

De las concentraciones plasmáticas peak de amikacina monitorizados, sólo $60 \%$ de ellas se encontraban en el rango objetivo de valores 10 veces sobre CIM, pero en $84,6 \%$ de los casos se mantuvo la dosis sin modificación; esta diferencia se explica porque hubo pacientes en que, aunque no se encontraban con concentraciones plasmáticas en rango óptimo, no se realizaron ajustes por su buena evolución clínica y/o recepción del resultado cerca del momento de la suspensión del fármaco. El 25\% de los pacientes que requirió ajuste fueron pacientes de oncología. Un estudio realizado en pacientes pediátricos con neutropenia febril postquimioterapia ${ }^{13}$ ya había evidenciado que las concentraciones plasmáticas peak alcanzadas con dosis de $15 \mathrm{mg} / \mathrm{kg}$ /día cada $24 \mathrm{~h}$ fueron suficientes en sólo $30 \%$ de ellos, mientras que los que recibieron la misma dosis fraccionada en dos veces al día sólo 8\% alcanzó concentraciones plasmáticas peak terapéuticas, mostrando la necesidad de monitorizar dichas concentraciones para lograr un ajuste individualizado de los esquemas de dosificación. En nuestra muestra, los pacientes oncológicos iniciaron tratamiento con 20 $\mathrm{mg} / \mathrm{kg}$ /día requiriendo ajuste en sólo $25 \%$ de los casos. Destaca que, de todos los pacientes sin comorbilidad, RN o de UCIP, en que se inició tratamiento con amikacina 15 $\mathrm{mg} / \mathrm{kg} /$ día, ninguno requirió ajuste de dosis, concordante con la recomendación actual generalizada de dosis para este antimicrobiano. Un análisis que puede inducir a error es que, al desglosar el tipo de paciente que alcanzaron concentraciones plasmáticas en rango terapéutico, 73,3\% fueron pacientes $F Q$ versus otro diagnóstico, pero esta situación no resultó estadísticamente significativa $(p=1)$ ya que responde netamente a la distribución de la muestra, donde la mayoría de pacientes eran portadores de FQ.

De las concentraciones plasmáticas valle de amikacina monitorizados, la mayoría $(84,6 \%)$ estuvo en rango 
esperado con valores $<1 \mu \mathrm{g} / \mathrm{mL}$, lo que demuestra que el iniciar tratamiento con dosis de $15 \mathrm{mg} / \mathrm{kg} /$ día y hasta $30 \mathrm{mg} / \mathrm{kg}$ /día es seguro. La mayoría de los estudios sobre monitorización terapéutica de amikacina hacen referencia a pacientes con características peculiares como RN, pacientes críticamente enfermos ${ }^{14}$ o pacientes quemados ${ }^{15,16}$ cuyos resultados de monitorización concuerdan en la necesidad de ajuste de dosis al iniciar terapia con dosis estándar de $15 \mathrm{mg} / \mathrm{kg}$ /día, información que no logramos replicar en nuestro trabajo.

Vancomicina es un antimicrobiano fundamental en el tratamiento de infecciones graves por bacterias grampositivas; su dosificación inadecuada se asocia con fracaso terapéutico, resistencia bacteriana y toxicidad ${ }^{17}$. La principal indicación de vancomicina fue la infección sanguínea (bacteriemias) o infecciones sin foco, pero con factor de riesgo, a pesar de que estos diagnósticos podrían determinar evoluciones graves, la mayoría de los tratamientos se realizó en sala básica.

Al efectuar la primera medición de Cbasal, la mayoría de los pacientes requirió ajustar dosis a pesar de haber iniciado tratamiento con la dosis recomendada de 60 $\mathrm{mg} / \mathrm{kg} /$ día. Ya en el año $2008^{18}$ se publicó una serie de 51 pacientes pediátricos que iniciaron tratamiento con vancomicina $40 \mathrm{mg} / \mathrm{kg} /$ día fraccionadas cada $12 \mathrm{~h}$ o $60 \mathrm{mg} / \mathrm{kg}$ /día fraccionado cada $6 \mathrm{~h}$, observando que en el primer grupo fueron necesarios mayores ajustes de dosis y frecuencia para obtener las concentraciones recomendadas.

La dispersión de Cbasal observada en nuestra población fue muy amplia, lo que se explica principalmente por el grupo de RN en quienes se utilizan dosis distintas a las de población general, ajustadas según edad cronológica y gestacional; además, en este grupo de pacientes se concentró el $25 \%$ de los ajustes realizados y el único paciente que requirió tres ajustes. La literatura científica avala la importancia de vancomicina en $\mathrm{RN}$ como tratamiento de sepsis de inicio tardío en presencia de cocáceas grampositivas; sin embargo, también confirma que la prescripción de la dosis correcta y el régimen de dosificación sigue siendo un desafío en las unidades de cuidados intensivos neonatales ${ }^{17}$. Según diversos trabajos en esta población, con la dosis de vancomicina recomendada actualmente, el objetivo terapéutico ABC/CIM > 400 se logra sólo en $25 \%$ de los pacientes ${ }^{19}$. El uso de concentraciones plasmáticas valle entre $10-20 \mu \mathrm{g} / \mathrm{mL}$ para reemplazar el ABC/CIM ha demostrado correlacionarse con este objetivo terapéutico ${ }^{20}$, razón por la que se usó sólo Cbasal de vancomicina en este estudio. Se han propuesto varios esquemas de dosificación de vancomicina, principalmente basados en la edad del RN (postconcepcional y postnatal), peso o creatininemia, sin lograr aún establecer consenso, por lo que un régimen de dosificación optimizado y monitorizado resulta esencial. Según nuestros datos, ninguno de los RN monitorizados alcanzó Cbasal dentro de rango terapéutico en la primera medición, lo que al compararlo con los resultados del resto de pacientes entrega un valor $\mathrm{p}=0,022$, lo que implica que al iniciar tratamiento con vancomicina en un $\mathrm{RN}$ es más probable que no se alcancen concentraciones plasmáticas terapéuticas, comparado con otros pacientes.

En nuestra muestra, la mayoría de los pacientes con FQ inició tratamiento con dosis estándar de vancomicina alcanzando concentraciones plasmáticas terapéuticas y en aquellos en que se modificó la dosis de inicio fue necesario el ajuste, lo que se contrapone con la publicación del año 2016 de McDade y cols. ${ }^{21}$, quienes analizaron un total de 90 tratamientos con vancomicina utilizando dosis de 60 $\mathrm{mg} / \mathrm{kg} /$ día observando que sólo $12,2 \%$ logró concentraciones plasmáticas terapéuticas, requiriendo dosis media de $70,6 \pm 16,7 \mathrm{mg} / \mathrm{kg} /$ día para lograr su objetivo; esta diferencia podría ser atribuible a la baja representatividad de pacientes con FQ tratados con vancomicina en nuestra muestra $(10,9 \%)$.

En los pacientes de UCIP estudiados, también se inició tratamiento con vancomicina $60 \mathrm{mg} / \mathrm{kg} / \mathrm{día}$ alcanzando la mayoría Cbasal en rango terapéutico en la primera medición, requiriendo ajuste sólo 20,8\% $(n=5)$ de ellos. En una serie de 81 pacientes pediátricos críticamente enfermos ${ }^{7}$ se observó que al utilizar dosis de $40 \mathrm{mg} / \mathrm{kg} /$ día, sólo $54 \%$ de ellos alcanzaba el parámetro objetivo ABC/CIM. Resultados similares se observan en un estudio nacional ${ }^{22}$ utilizando dosis promedio de vancomicina 40 $\mathrm{mg} / \mathrm{kg} / \mathrm{d}$ ía, en que sólo lograron en $40 \%$ de los casos Cbasales mayores a $10 \mu \mathrm{g} / \mathrm{mL}$; los autores concluyeron que probablemente era necesario iniciar terapias con dosis mayores y efectuar seguimiento con monitorización de concentraciones plasmáticas. Del mismo modo, en otra publicación que evaluó 22 pacientes $^{23}$ que iniciaron tratamiento con vancomicina $40 \mathrm{mg} / \mathrm{kg} /$ día, sólo $50 \%$ de ellos alcanzó ABC/CIM > 400. Ambas publicaciones reafirman la dosis de $60 \mathrm{mg} / \mathrm{kg} /$ día como la más adecuada para el inicio de terapia.

\section{Conclusión}

Nuestro estudio confirma la necesidad de monitorización terapéutica de amikacina y/o vancomicina en pacientes pediátricos y particularmente en $\mathrm{RN}$ o pacientes con FQ, en quienes la monitorización permite ajustar dosis y alcanzar metas terapéuticas en forma individualizada, disminuyendo la probabilidad de fracasos terapéuticos o emergencia de resistencias bacterianas. Nuestros resultados corroboran la importancia de iniciar tratamiento con amikacina $15 \mathrm{mg} / \mathrm{kg} /$ día en pacientes en general, excepto en aquellos con FQ, quienes necesitan dosis mayores; y vancomicina $60 \mathrm{mg} / \mathrm{kg} /$ día para tener mayor probabilidad 
comicina donde la mayoría de los pacientes eran sanos, pudiendo ser el análisis de sub-poblaciones oncológicas y FQ poco representativo. Existen pocos estudios en población pediátrica que analicen la monitorización de antimicrobianos, particularmente en pacientes con FQ y oncológicos, por lo que este trabajo sirve como una primera aproximación para describir si las dosis indicadas en estas poblaciones alcanzan concentraciones plasmáticas terapéuticas. Serán necesarios estudios prospectivos en población pediátrica bien definida para poder corroborar las dosis empíricas iniciales y representar de mejor manera las poblaciones con comorbilidad y $\mathrm{RN}$.

\section{Referencias bibliográficas}

1.- Nichols K. Pediatric Antimicrobial Stewardship Programs. J Pediatr Pharmacol Ther. 2017; 22 (1): 77-80. doi: 10.583/1551-6776-22.1.77.

2.- Zylbersztajn B, Barraza M, Torres J, Morales J. Monitorización terapéutica de antimicrobianos en pediatría. Revisión de la experiencia latinoamericana. Rev Chilena Infectol. 2018; 35 (1): 22-8. http://dx.doi.org/10.4067/s071610182018000100022.

3.- Cisneros J, Neth O. Global impact of an educational antimicrobial stewardship programme on prescribing practice in a tertiary hospital centre. Clin Microbiol Infect. 2014; 20 (1): 82-8. doi: 10.1111/1469-0691.12191.

4.- Ritz N, Bielicki J, Pfister M, Van der Anker J. Therapeutic drug monitoring for anti-infective agents in pediatrics. The way forward. Pediatr Infect Dis J. 2016; 35 (5): 570-2. doi: 10.1097/ INF.0000000000001091.

5.- Liu C, Bayer A, Cosgrove S, Daum R S, Fridkin S K, Gorwitz R J, et al; Infectious Diseases Society of America. Clinical Practice Guidelines by the Infectious Diseases Society of America for the Treatment of MethicillinResistant Staphylococcus aureus Infections in Adults and Children. Clin Infect Dis. 2011; 52: e18-e55. doi: 10.1093/cid/ciq146.

6.- Hernández R. Pharmacokinetics and pharmacodynamics of antibiotics used in pediatric patients with cystic fibrosis. Neumol Pediatr. 2016; 11 (1): 23-7. Disponible en: https://www.neumologia-pediatrica.cl/ revista/2016-11-1/.

7.- Acuña C, Morales J, Castillo C, Torres J. Farmacocinética de vancomicina en niños hospitalizados en una unidad de paciente crítico. Rev Chilena Infectol. 2013; 30 (6): 585-90. http://dx.doi.org/10.4067/S071610182013000600001.

8.- Ku L, Smith P. Dosing in neonates: special considerations in physiology and trial design. Pediatr Res. 2015; 77 (1-1): 2-9. doi: 10.1038/ pr.2014.143.
9.- Contopoulos-Ioannidis D, Giotis N, Baliatsa D, Ioannidis J P A. Extended-interval aminoglycoside administration for children: a meta-analysis. Pediatrics. 2004; 114 : e111-8. http://dx.doi.org/10.4067/S071610182004000400011

10.- Schwartz G J, Muñoz A, Schneider M F, Mak R H, Kaskel F, Warady B A, et al. New equations to estimate GFR in children with CDK. J Am Soc Nephrol. 2009; 20: 629-37. doi: 10.1681 / ASN.2008030287.

11.- Young T E, Magnum B. Neofax 2010: A manual of drugs used in neonatal care. Ed 23. Montvale, NJ. Thompson Reuters Clinical Editorial. 2010.

12.- Hoban D, Bouchillon S, Hawser S, Badal R, Labombardi V, DiPersio J. Susceptibility of gram-negative pathogens isolated from patients with complicated intraabdominal infections in the United States, 2007-2008: Results of the Study for Monitoring Antimicrobial Resistance Trends (SMART). Antimicrob Agents Chemother. 2010; 54 (7): 3031-4. doi: 10.1128 / AAC.01808-09

13.- Rabello M, Villena R, Morales J, Aravena R, Kopp K, Villarroel M, et al. Pharmacokinetics of amikacin in children with cancer and febrile neutropenia. Poster, 49th Annual Meeting of the Infectious Diseases Society of America (IDSA), October 20-23, 2011. https://idsa.confex.com/ idsa/2011/webprogram/Paper32323.html

14.- Bressolle F, Gouby A, Martínez J, Joubert P, Saissi G, Guillaud R, et al. Population pharmacokinetics of amikacin in critically ill patients. Antimicrob Agents Chemother. 1996; 40 (7): 1682-9. doi:10.1128/AAC.40.7.1682.

15.- Kopcha R, Fant W, Warden G. Increased dosing requirements for amikacin in burned children. J Antimicrob Chemother. 1991; 28 (5): 747-52. doi: 10.1093 / jac / 28.5.747.

16.- Sherwin C, Wead S, Stockmann C, Healy D, Spigarelli M G, Neely A, et al. Amikacin population pharmacokinetics among paediatric burn patients. Burns. 2014; 40 (2): 311-8. doi: 10.1016 / j.burns.2013.06.015.
17.- Monteiro J, Hahn S, Gonçalves J, Fresco P. Vancomycin therapeutic drug monitoring and population pharmacokinetic models in special patient subpopulations. Pharmacol Res Perspect. 2018; e004-20. doi: 10.1002/ prp2.420.

18.- Zylbersztajn B, Travaglianti M, Weller G, Mato $\mathrm{H}$. Monitoreo terapéutico de vancomicina en una terapia intensiva pediátrica. Arch. Argent. Pediatr.2008; 106 (1): 26-31.

19.- Padari H, Oselin K, Tasa T, Metsvaht T, Loivukene K, Lutsar I. Coagulase negative staphylococcal sepsis in neonates: do we need to adapt vancomycin dose or target? BMC Pediatr. 2016; 16: 206. https://doi.org/10.1186/ s12887-016-0753-0.

20.- Rybak M, Le J, Lodise T, Levine D P, Bradley J S, Liu C, et al. Therapeutic monitoring of vancomycin for serious methicillinresistant Staphylococcus aureus infections: A revised consensus guideline and review by the American Society of Health-System Pharmacists, the Infectious Diseases Society of America, the Pediatric Infectious Diseases Society, and the Society of Infectious Diseases Pharmacists. Am J Health Syst Pharm. 2020; 77: 835-64. doi: 10.1093/ajhp/zxaa036.

21.- McDade E, Hewlett J, Baker C. Evaluation of vancomycin dosing in pediatric cystic fibrosis patients. J Pediatr Pharmacol Ther. 2016; 21 (2): 155-61. doi: 10.5863 / 1551-6776-21.2.155.

22.- Villena R, González C, Nalegach M E, Vásquez A, Villareal M, Drago M. Monitoreo terapéutico de vancomicina intravenosa en una unidad de paciente crítico pediátrico. Rev Chilena Infectol. 2014; 31 (3): 249-53. Disponible en: https://scielo.conicyt.cl/pdf/rci/ v31n3/art01.pdf.

23.- Giachetto G, Telechea H, Speranza N, Oyarzún M, Nanni L, Menchaca A. Vancomycin pharmacokinetic pharmacodynamic parameters to optimize dosage administration in critically ill children. Pediatr Crit Care Med. 2011; 12 (6): e250-4. doi: 10.1097 / PCC.0b013e3181fe4047. 